\title{
Photonic magnetic metamaterial basics
}

\author{
Kamil Boratay Alici ${ }^{\mathrm{a}, \mathrm{b}, *}$, Andriy E. Serebryannikov ${ }^{\mathrm{a}, \mathrm{c}}$, Ekmel Ozbay ${ }^{\mathrm{a}, \mathrm{b}, \mathrm{d}}$ \\ ${ }^{a}$ Nanotechnology Research Center, Bilkent University, Bilkent, 06800 Ankara, Turkey \\ ${ }^{\mathrm{b}}$ Department of Physics, Bilkent University, Bilkent, 06800 Ankara, Turkey \\ ${ }^{\mathrm{c}}$ Hamburg University of Technology, D-21071 Hamburg, Germany \\ ${ }^{\mathrm{d}}$ Department of Electrical and Electronics Engineering, Bilkent University, Bilkent, 06800 Ankara, Turkey
}

Received 20 April 2010; received in revised form 3 June 2010; accepted 9 July 2010

Available online 21 July 2010

\begin{abstract}
In the present study, we provide a detailed analysis for the study of photonic metamaterials. We demonstrate the polarization and orientation dependent transmission response of split ring resonators at the infrared and visible band. We provided optical measurements only for one case, in which electric component of the incident field was coupled to planar split ring resonator array. We consecutively studied (i) frequency tuning, (ii) effect of resonator density, (iii) shifting magnetic resonance frequency by changing the resonator shape, and (iv) effect of metal loss and plasma frequency. The study provides an overlook for the candidate applications such as the enhancement of power passing through an electrically small hole, negative index metamaterials and optical metamaterial absorbers.
\end{abstract}

(C) 2010 Elsevier B.V. All rights reserved.

Keywords: Photonic metamaterial; Tunability; Magnetic resonance; Split ring resonator

\section{Introduction}

Artificially constructed periodic metamaterials that exhibit unusual properties such as simultaneous negative permeability and negative permittivity that are not present in nature have attracted considerable interest [114]. The first feasible example of negative permeability medium was composed of microstructures built from nonmagnetic conducting sheets [1]. One of the first significant applications of these structures was to manipulate the RF flux in all nuclear magnetic resonance systems [2]. Transmission and reflection based characterization of double negative medium that simulta-

\footnotetext{
* Corresponding author at: Nanotechnology Research Center, Bilkent University, Bilkent, 06800 Ankara, Turkey.

E-mail address: bora@fen.bilkent.edu.tr (K.B. Alici).
}

neously has negative permeability and negative permittivity was provided at microwave regime [3]. The physical size of their unit cell size determines the operation frequency and it is typically an order of magnitude smaller than the operation wavelength [4]. The unit cell geometry can be optimized to miniaturize metamaterial elements yielding small and strongly resonant inclusions $[4,14]$. Negative index of metamaterials can be tested by measuring flat and prism shaped metamaterial media with oblique and normal incidence transmission measurements respectively [5]. Planar double negative metamaterials were also demonstrated for normal incidence and oblique incidence at the millimeter-wave frequencies $[6,15]$. At the $\mathrm{THz}$ regime active metamaterials provided efficient real-time control and manipulation of radiation [7]. Moreover, nonlinear magneto-inductive waves in left-handed composite metamaterials were demonstrated theoretically [12]. 
As a practical application, metamaterial inspired small radiation sources were demonstrated and their fundamental radiation limitations were studied [11].

Metamaterials are shown to operate at microwave [10], millimeter-wave [5,6], THz [7], mid-IR [9], IR and visible [8] regimes of electromagnetic spectrum. Utilization of metamaterials at the optical regime could lead to high performance and novel photonic devices in information and communication technologies (ICT). It is possible to implement negative index material based superlenses, optical isolators, electro-optic modulators, and optical switches [13]. In the present paper, we present basic analysis of photonic metamaterials, especially focusing on their magnetic response. We provide a complete study, which starts with nanofabrication, optical simulation method, and experimental characterization and continue with detailed discussion of polarization dependence, effect of buffer layer thickness, density, and intrinsic metal properties.

\section{Methodology}

In this part, we explain our design simulations, nanofabrication techniques, and optical measurement setups.

\subsection{Design simulations}

We used the commercial software CST-Microwave Studio [16], which uses the full wave finite integration method, while analyzing the magnetic metamaterial unit cells. We assumed the unit cell to be infinitely periodic at the lateral directions and used the program's frequency domain solver with unit cell boundary conditions. Metamaterials composed of shaped metallic resonators placed on a substrate. We used experimental data to estimate their actual parameters such as metal loss, coated metal thickness, resonator side length, strip width, and arm length of these structures. We also utilized from the literature data for the metal characteristics. During the fabrication step we paid specific attention to achieve the structure parameters as designed.

\subsection{Nano-fabrication}

The first step was the preparation of the sapphire $\left(\mathrm{Al}_{2} \mathrm{O}_{3}\right)$ substrate. It was diced to $7 \mathrm{~mm} \times 7 \mathrm{~mm}$ dimensions, cleaned in acetone, isopropanol, deionized water consecutively, and dried using a nitrogen gas gun. The sample was placed on a hot-plate at $180{ }^{\circ} \mathrm{C}$ for $1 \mathrm{~min}$ for dehydration. Then, we coated it with polymethyl methacrylate (PMMA-950K-A2), a high resolution positive resist, via a spinner machine to achieve a
$150 \mathrm{~nm}$ thickness and baked it at $180{ }^{\circ} \mathrm{C}$ for $90 \mathrm{~s}$. In order to prevent charging during e-beam exposure we used aquasave coating. The e-beam exposure step starts with the design of the exposure structures and dose tests.

We drew the SRRs as a composition of single pixel lines using the Raith e-beam lithography computer aided controller program. For the dose tests, a twodimensional array of SRRs with slightly varying parameters was organized. The acceleration voltage was $15 \mathrm{kV}$. The next step, development, took $45 \mathrm{~s}$ with a solution of 1:3 ratio MIBK: $\mathrm{H}_{2} \mathrm{O}$. Then, the sample was coated with silver $(\mathrm{Ag})$ via an e-beam evaporator machine. The final steps were the lift-off and scanning electron microscopy (SEM) inspection. After determining the optimum dose, we fabricated the SRR array coated sample, as shown in Fig. 1.

\subsection{Experiment}

The transmission measurements of the samples were performed using a homemade transmission setup. We used an Ocean Optics LS-1 tungsten halogen light source. Light was passing through a Glan Taylor cube polarizer and focused on the SRR printed area via a Melles-Griot $20 \times$ objective. The beam diameter was around $100 \mu \mathrm{m}$. We collected the light with a $50 \times$ objective and by the aid of a mirror we sent the beam to a CCD camera. We found the position of the SRR array on the sapphire sample and then centered the light beam on that area. After alignment we allowed the light to go to the lens and multimode fiber. The signal was measured using an Ocean Optics USB4000 spectrometer that operates at the visible regime. We calibrated the sample's response with respect to bare substrate. The spectrometer can measure the 600-1000 nm band. In our test measurements, we characterized several SRR arrays with different parameters and observed the shift of electrical resonance as was expected.

\section{Results and discussions}

We had to find a method that can take into account the fabrication imperfections. For the e-beam lithography fabrication technique, the resonator parameters can be different from the designed ones on the order of a few nm. SEM image precision limit should also be taken into account. We observed in SEM inspections that for each of the single SRR unit, we had a different set of parameters. Each SRR was actually different than the others. The parameters varied at most $5 \mathrm{~nm}$ from one SRR side length to another at different regions of the array. Our solution was to find out a set of average SRR 


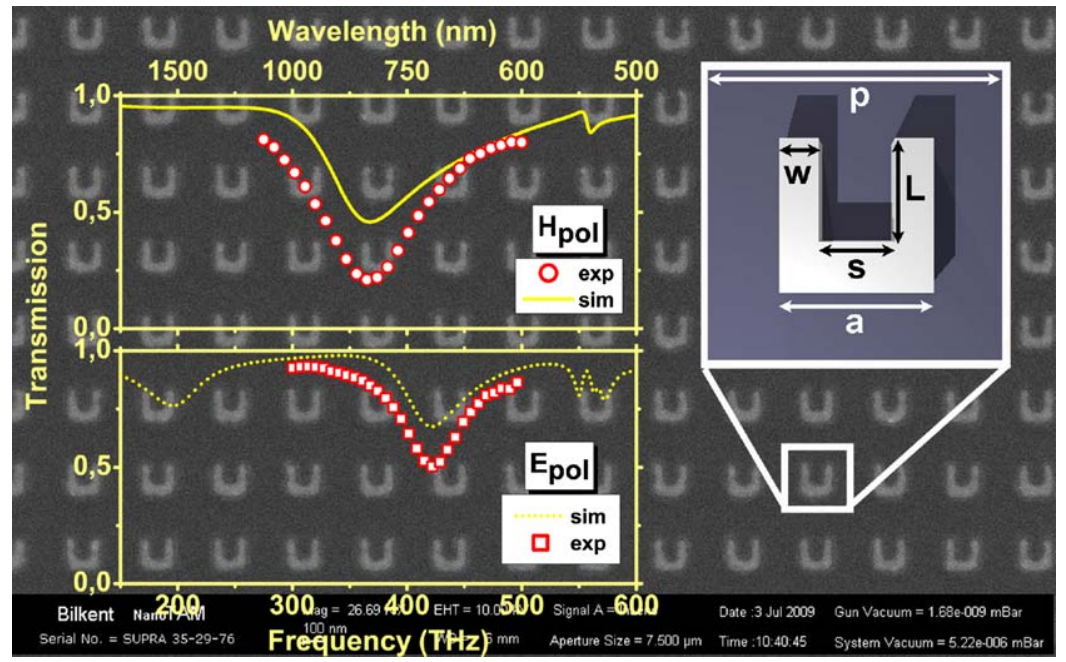

Fig. 1. Scanning electron microscopy image of the fabricated array (background). Simulated and measured transmission responses (left). Schematic and parameters of the unit cell (right).

parameters that gave the same response as the experimentally characterized one. The unit cell of the average SRR whose transmission response is close to the measured SRR array is shown in Fig. 1. In the same figure, we also show the simulation and experiment results for the configuration that is to be studied in detail in the following section. The periods in the $x$ and $y$ directions were $p_{x}=p_{y}=330 \mathrm{~nm}$, side length of the SRR at the $x$ and $y$ directions were $a_{x}=a_{y}=155 \mathrm{~nm}$, arm length was $L=104 \mathrm{~nm}$, width of the arms was $w=39 \mathrm{~nm}$, separation between the arms was $s=77 \mathrm{~nm}$ and the coated metal thickness was $h=39.5 \mathrm{~nm}$. We took the substrate thickness in the simulations as $t=150 \mathrm{~nm}$ while it was $1 \mathrm{~mm}$ in the experiments. In the simulation domain, we observed that using a substrate thickness larger than $150 \mathrm{~nm}$ does not change the resonance frequency of the SRR array. Thereby, in all of our simulations, we used a $150 \mathrm{~nm}$ thick substrate instead of a $1 \mathrm{~mm}$ one. At the optical regime, the dispersion characteristics of metals play an important role. They were taken into account by fitting the complex metal permittivity values given in Palik [17] using the Drude model. CST Drude model permittivity was given by the formula: $\varepsilon(\omega)=\varepsilon_{\infty}+\omega_{p}^{2} / \omega\left(\omega-i v_{c}\right)$ where $\omega_{p}$ was the plasma frequency and $v_{c}$ was the collision frequency. Our fit to the Palik data implied $\omega_{p}=13,250 \times 10^{12} \mathrm{rad} / \mathrm{s}$ and $v_{c}=130 \mathrm{THz}$. We used this data set to model the dispersion properties of silver in our simulations. However, we discovered from the experimental data that the collision frequency of our metal was 4.3 times larger. Despite this, we used the values of $\omega_{p}$ and $v_{c}$ obtained from the fit of Palik data in the following theoretical analysis.

\subsection{Transmission response depends on the incident field polarization and unit cell orientation}

The significance of SRR relies on its magnetic resonance. Depending on the orientation of the SRR and polarization of the incident light we could excite the magnetic resonance of the SRR by E-field, B-field, or both E- and B-field of the incident light. It is also possible that the SRR may not be excited at all. In Figs. 2 and 3, we show the possible orientations and corresponding transmission response of the SRRs. For the planar case, as shown in Fig. 2 as the (blue) dotted curve, we observed two transmission stop bands. The first one appeared due to the magnetic resonance, and the second one appeared due to the electrical resonance

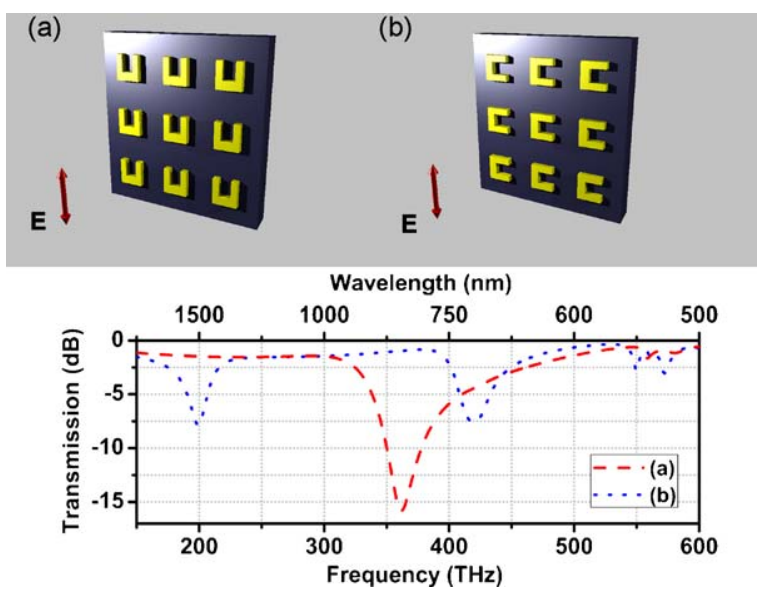

Fig. 2. Different orientations and transmission response of the SRR medium (a) only the electric resonance was excited, (b) both electric and magnetic resonances were excited. 


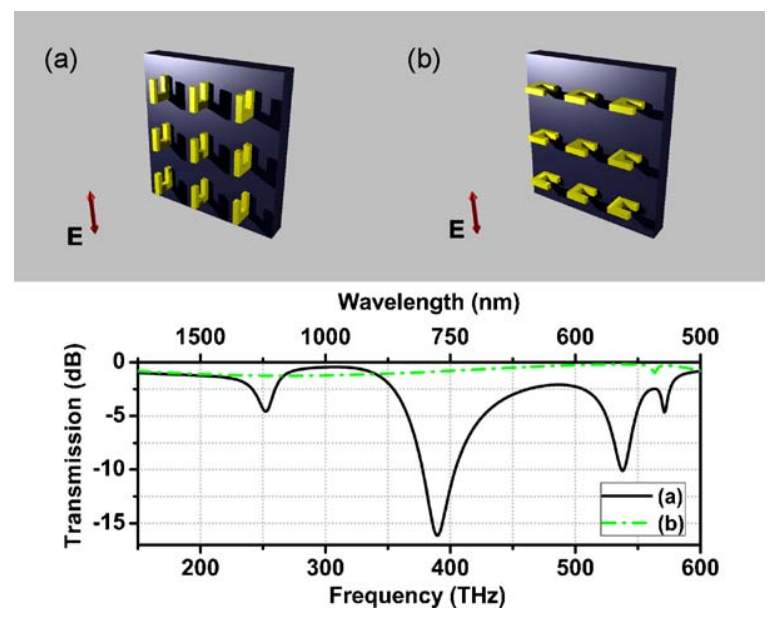

Fig. 3. Other possible orientations and transmission response of the SRR medium: (a) both electric and magnetic resonances were excited by the B-field of the incident wave, (b) none of the resonances were excited.

of the SRR. At the magnetic resonance, we had circulating currents at the SRRs, so that they acted like magnetic dipoles. This stop band was at around $1550 \mathrm{~nm}$ wavelength. The SRRs physical size was an order of magnitude smaller than this operation wavelength. At the electrical resonance mode, we observed an electric dipole like response and the stop band was around $700 \mathrm{~nm}$ wavelength. For the other orientation shown in Fig. 2b, we could only obtain the electrical resonance since the magnetic resonance of the structure was not excited. We saw a stop band at around $830 \mathrm{~nm}$ that was slightly different from case (a). We have not yet obtained a coupling effect between the two modes (magnetic and electric) as in case (a). By this analysis, we can characterize our SRRs and determine the operation frequency that corresponds to the magnetic resonance frequency. In Fig. 3, we show the other possible orientations of the SRRs with respect to the incident field. The magnetic resonance frequency for this case was around $1200 \mathrm{~nm}$ and the electrical resonance was at $770 \mathrm{~nm}$. In case (a), the magnetic resonance was excited by the B-field of the incident wave and in case (b) none of the resonances were excited.

\subsection{Resonance wavelength shifts in parallel with the thickness of a dielectric layer between the substrate and SRRs}

Based on the simulation results we developed a method for the fine tuning of the magnetic and electric resonances of SRR arrays. When the substrate itself was

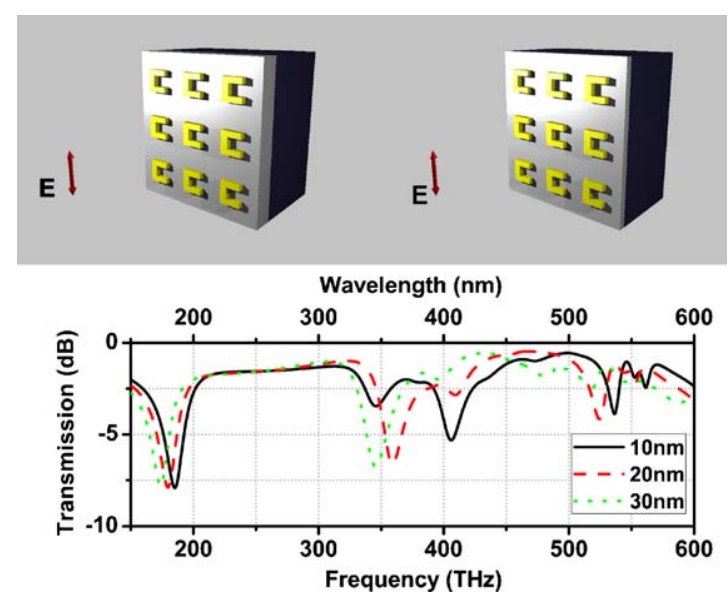

Fig. 4. Effect of changing buffer layer thickness on the magnetic resonance frequency.

very thin, or coated with a very thin buffer layer, the magnetic resonance frequency shifts, depending on the layer thickness. In Fig. 4, we presented the results for the glass layer of thicknesses varying from 10 to $30 \mathrm{~nm}$. As this buffer layer thickens, the resonance frequency shifts from $185.1 \mathrm{THz}$ to $173.8 \mathrm{THz}$. As we continue to increase the buffer layer $\left(t_{b}\right)$ or substrate thickness $(t)$, the shift shows a saturating behavior and does not change at around $150 \mathrm{~nm}$ thickness. Using materials whose volume is dependent on the applied temperature, pressure, or static field, we can slightly tune the metamaterial resonance frequency.

We tried to explain some of the effects observed here using the theory of perturbations [18]. For small buffer layer thickness, or small substrate thickness without buffer a layer, the shift of the resonance frequency can be qualitatively explained by considering the effect of substrate as a perturbation of the resonance field of the substrate-free SRR. Indeed, it is well known from the theory of perturbations of the closed cavities with a perfect electric conductor (PEC) wall that introducing a dielectric perturbation into such a cavity leads to a downshift of the eigenfrequencies. In this case, the perturbation affects the electric field. Indeed, in the general case only the frequency shift can be estimated as $\Delta f / f=-\left(\varepsilon-\varepsilon_{0}\right) F / \Omega-\left(\mu-\mu_{0}\right) G / \Omega$, where $\varepsilon_{0}$ and $\mu_{0}$ are free-space permittivity and permeability, $\varepsilon$ and $\mu$ are permittivity and permeability of the perturbation, $f$ is an eigenfrequency of the unperturbed cavity and $\Omega$ is the corresponding eigenmode energy; $F$ and $G$ are the variations of the energy of the electric and magnetic fields arising due to the introduced perturbation. Thus the second term is vanishing if $\mu=\mu_{0}$. Analogously, placing a dielectric substrate below a 
SRR in turn results in the enhancement of the resonance electric field, which in turn increases the effective capacitance and, hence, decreases the magnetic resonance frequency $\left(f_{m}\right)$. Furthermore, using this analogy one could expect that placing a diamagnetic $\left(\mu<\mu_{0}\right)$ or low-permittivity $\left(\varepsilon=\varepsilon_{0}\right)$ substrate instead of a dielectric one should result in the upshifting of $f_{m}$. Further increase of the substrate thickness leads to the saturation of $f_{m}$. In other words, it does not lead to any significant variation in $f_{m}$ starting from a certain value of $t_{b}$ of $t$. This is quite predictable since only the nearinterface part of the substrate perturbatively affects the resonance field of the substrate-free SRR. Our perturbation theory based interpretation is in agreement with the results presented in a Wegener Group paper, see Fig. 2 in Ref. [19]. Therein, the increase of the index of refraction of the dielectric substrate material, $n$, results in the downshifting of $f_{m}$. For larger $n$ values, we obtain a stronger perturbation of the resonance electric field.

\subsection{Resonance bandwidth is inversely proportional to the density of split ring resonators}

In Fig. 5, we demonstrated the effect of the SRR period on the resonant behavior. We present the cases when the period was $1.4,2,4$, and 6 times the side length of the SRR. As the period was increased, we observed two significant features. First, due to the weakened coupling of the SRRs, the fractional bandwidth of the stop band decreased. Moreover, the resonance strength reduced while the resonance frequency decreased. The second outcome was that the response of the SRR array nearly became the same as the single SRR as the period increased up to $6 a$.

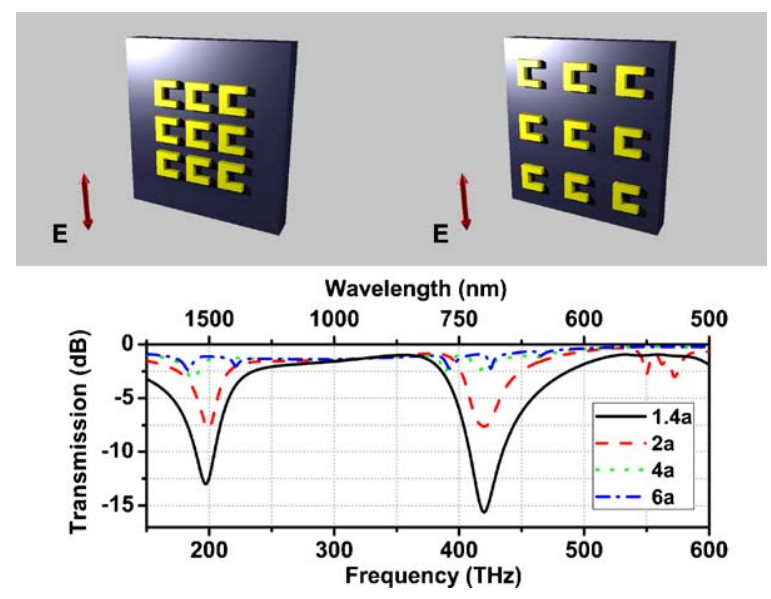

Fig. 5. Effect of SRR period.
Therefore, in our future studies in order to observe the single resonator effects, we could also use periodic SRRs with a sufficiently large period.

\subsection{Resonator arm length is critical for the magnetic resonance frequency}

One of the most important parameters of photonic SRRs that determine the magnetic resonance frequency was the arm length $L$. In Fig. 6, we decreased the arm length from $104 \mathrm{~nm}$ to $0 \mathrm{~nm}$ and observed a quite strong shift of magnetic resonance frequency. The electrical resonance remained nearly the same while the magnetic resonance shifts from $199.5 \mathrm{THz}$ to $324.6 \mathrm{THz}$ when the arm length reduced from $104 \mathrm{~nm}$ to $35 \mathrm{~nm}$. The limiting case, $L=0$, corresponds in fact to the square particles. In this case, the magnetic resonance approached its limit and we only had the electrical resonance present. In this part, we observed that the reduction of the arm length increases the operation frequency, but the electrical size of the SRRs also reduced and they were not in the deep subwavelength regime any more. Therefore, the array moved from the metamaterial regime to the photonic crystal regime.

The analogy with the perturbed PEC-wall cavities can again be adopted, since $\omega_{p}$ is still several times larger than $2 \pi f_{m}$. Accordingly, the variation of the SRR shape from $L=104 \mathrm{~nm}$ to $0 \mathrm{~nm}$ can be qualitatively interpreted as a perturbation due to the introduction of a PEC body into the cavity. In this case, the frequency shift is $\Delta f / f=-\varepsilon_{0} F / \Omega+\mu_{0} G / \Omega$ [18]. Hence, such a perturbation should lead to the upshift of the resonance frequency, if the effect of the magnetic field dominates over that of the electric field. According to recent studies by the Soukoulis group [20,21], the magnetic

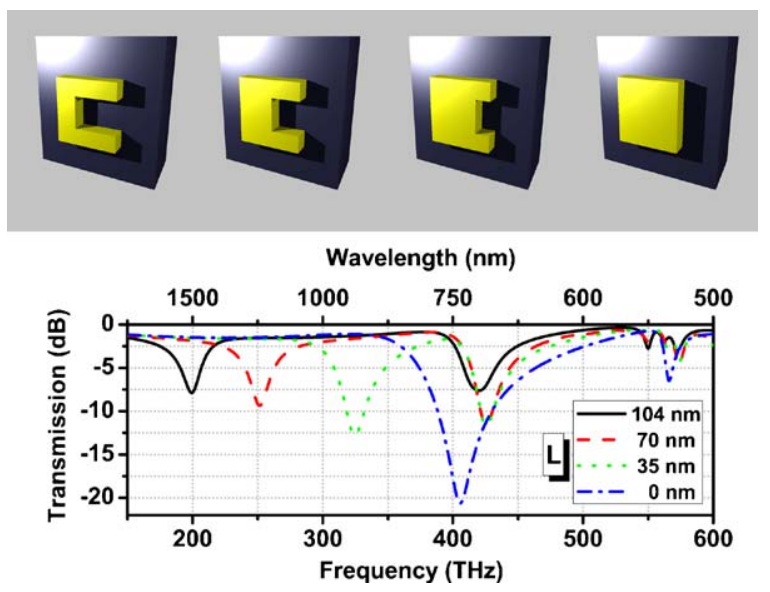

Fig. 6. Effect of changing the arm length $L$. 
resonance frequency can be extracted from a quasistatic LC-circuit theory in the following form: $f_{m}=(1 / 2 \pi)\left(1 / \sqrt{\left(L_{e}+L_{m}\right) C}\right)$, where $L_{e}$ is the electron self-inductance related to the kinetic energy, $L_{m}$ is the magnetic field inductance, and $C$ is capacitance of the split ring. In turn, both $L_{e}$ and $L_{m}$ are proportional to the length of the axis of the wire making the ring, $l^{\prime}$. The structures shown in Fig. $6 l^{\prime}$ decreased from left to right leading to larger $f_{m}$. Hence, the results of the qualitative analysis based on both perturbation and LC-circuit interpretations are in agreement.

\subsection{Low loss metal leads to higher resonance frequency}

As the operation frequency of the metamaterials increase, the effect of plasma frequency of the metal cannot be neglected. A metal with either a larger or smaller plasma frequency $\left(\omega_{p}\right)$ than that of silver $(\mathrm{Ag})$ enables one to obtain larger or smaller values of $f_{m}$ compared to silver. In the above formula for $f_{m}[21], \omega_{p}$ appears due to the term $L_{e} \propto \omega_{p}^{-2}$. In the hypothetic limiting case, $L_{m}=0, f_{m} \propto \omega_{p}$ and the contribution of $L_{e}$ vanishes if $\omega_{p}$ tends to infinity. Silver $(\mathrm{Ag})$ is one of the low loss metals that has also been demonstrated to be less lossy than gold $(\mathrm{Au})$ and antimony $(\mathrm{Pb})$ [22]. The high frequency response of SRR can be achieved by modifying the SRR shape. We can obtain a magnetic resonance at higher frequencies with the same SRR side length, but by varying the arm length as shown in the previous part. However, we should keep in mind that high frequency operation is then achieved at the expense of electrical size. Another suggestion was to use metals

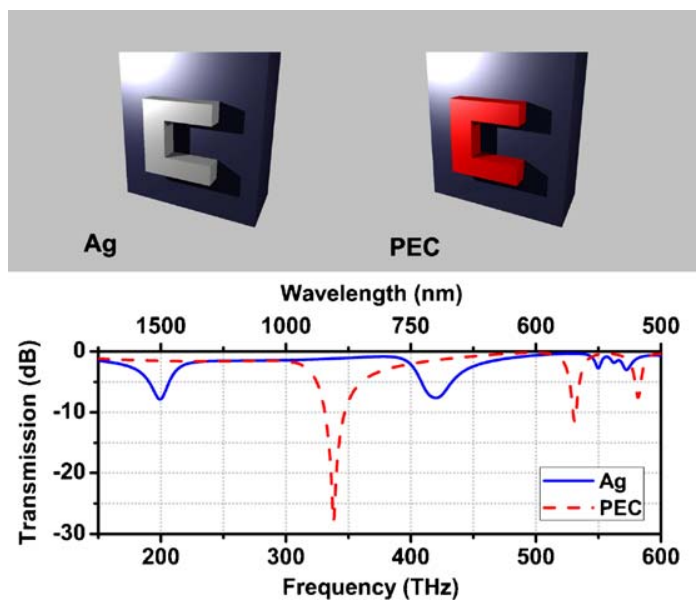

Fig. 7. Effect of the metal loss and plasma frequency of the SRR material. at low temperatures to be able to push the resonant behavior at higher frequencies while keeping the electrical size the same [23]. In Fig. 7, we show how our resonator would behave if we had the opportunity to fabricate it from a perfect electrical conductor. The stop band of the magnetic resonance moved from $199.5 \mathrm{THz}$ to $338.1 \mathrm{THz}$, and the resonance strength changed from $-8 \mathrm{~dB}$ to $-28 \mathrm{~dB}$ while replacing the PEC SRRs with the silver ones. However, even with the PEC SRR, the metamaterial operation cannot cover the entire visible spectrum due to the fundamental limitations of the used SRR designs.

\section{Conclusion}

We clearly demonstrated the possible effects of split ring resonator orientations on the transmission response at the optical regime. Depending on the application, a different orientation and correspondingly different modes can be utilized. When the thickness of the substrate on which the split ring resonators were printed was very small, it may affect the magnetic resonance frequency. We can mimic this behavior by coating a thick substrate with a very thin buffer layer. The magnetic resonance frequency can be fine tuned by changing the buffer layer thickness. For a densely packed split ring resonator array, a stronger coupling yielded an increased the fractional bandwidth of the magnetic stop band, as well as an increased the resonance strength. When the split ring resonators were loosely packed, the response was very weak and very similar to that in a single resonator case. The magnetic resonance frequency was strongly dependent on the parameter: arm length $(L)$ of the split ring resonators. A slight change in the arm length leads to a significant shift of the magnetic resonance frequency. The resonant behavior of metamaterials at the optical regime strongly depends on the characteristics of the utilized metal. There was a $139 \mathrm{THz}$ difference between the magnetic resonance frequencies of silver- and perfect electrical conductor-based split ring resonator media. These results altogether provide the basics of the photonic metamaterial study and they will be quite useful for the applications.

\section{Acknowledgements}

This work is supported by the European Union under the projects EU-PHOME, and EU-ECONAM, and TUBITAK under the project nos., 107A004, and 107A012. One of the authors (E.O.) also acknowledges partial support from the Turkish Academy of Sciences. 


\section{References}

[1] J.B. Pendry, A.J. Holden, D.J. Robbins, W.J. Stewart, Magnetism from conductors and enhanced nonlinear phenomena, IEEE Trans. Microwave Theor. Tech. 47 (1999) 2075-2084.

[2] M.C.K. Wiltshire, J.B. Pendry, I.R. Young, D.J. Larkman, D.J. Gilderdale, J.V. Hajnal, Microstructured magnetic materials for RF flux guides in magnetic resonance imaging, Science 291 (2001) 849-851.

[3] E. Ozbay, K. Aydin, E. Cubukcu, M. Bayindir, Transmission and reflection properties of composite double negative metamaterials in free space, IEEE Trans. Antennas Propag. 51 (2003) 25922595.

[4] K.B. Alici, F. Bilotti, L. Vegni, E. Ozbay, Miniaturized negative permeability materials, Appl. Phys. Lett. 91 (2007) p071121.

[5] K.B. Alici, E. Ozbay, Theoretical study and experimental realization of a low-loss metamaterial operating at the millimeterwave regime: demonstrations of flat and prism shaped samples, IEEE J. Select. Top. Quant. Electron. 16 (2010) 386-393.

[6] K.B. Alici, E. Ozbay, Characterization and tilted response of a fishnet metamaterial operating at $100 \mathrm{GHz}$, J. Phys. D: Appl. Phys. 41 (2008) p135011.

[7] H.-T. Chen, W.J. Padilla, J.M.O. Zide, A.C. Gossard, A.J. Taylor, R.D. Averitt, Active terahertz metamaterial devices, Nature 444 (2006) 597-600.

[8] C. Enkrich, M. Wegener, S. Linden, S. Burger, L. Zschiedrich, F. Schmidt, J.F. Zhou, Th. Koschny, C.M. Soukoulis, Magnetic metamaterials at telecommunication and visible frequencies, Phys. Rev. Lett. 95 (2005) p203901.

[9] N. Katsarakis, G. Konstantinidis, A. Kostopoulos, R.S. Penciu, T.F. Gundogdu, M. Kafesaki, E.N. Econoumou, Th. Koschny, C.M. Soukoulis, Magnetic response of split-ring resonators in the far-infrared frequency regime, Opt. Lett. 30 (2005) 1348-1351.

[10] L. Zhang, G. Tuttle, C.M. Soukoulis, GHz magnetic response of split ring resonators, Photon. Nanostruct. 2 (2004) 155-159.
[11] K.B. Alici, E. Ozbay, Electrically small split ring resonator antennas, J. Appl. Phys. 101 (2007) 083104.

[12] I. Shadrivov, A. Zharov, N. Zharova, Y. Kivshar, Nonlinear magnetoinductive waves and domain walls in composite metamaterials, Photon. Nanostruct. 4 (2006) 69-74.

[13] D.R. Smith, J.B. Pendry, M.C.K. Wiltshire, Metamaterials and negative refractive index, Science 305 (2004) 788-790.

[14] K.B. Alici, F. Bilotti, L. Vegni, E. Ozbay, Optimization and tunability of deep subwavelength resonators for metamaterial applications: complete enhanced transmission through a subwavelength aperture, Opt. Express 17 (2009) 5933-5943.

[15] K.B. Alici, E. Ozbay, A planar metamaterial: polarization independent fishnet structure, Photon. Nanostruct. 6 (2008) 102-107.

[16] CST, GmbH, CST-Microwave Studio, Darmstadt, Germany, 2009.

[17] E.D. Palik, Handbook of Optical Constants of Solids, Academic Press, San Diego, 1998.

[18] V.V. Nikolskiy, Theory of Electromagnetic Field, Vysshaya Shkola Press, Moscow, 1964.

[19] M. Husnik, M.W. Klein, N. Feth, M. Konig, J. Niegemann, K. Busch, S. Linden, M. Wegener, Absolute extinction cross-section of individual magnetic split-ring resonators, Nat. Photon. 2 (2008) 614-617.

[20] C.M. Soukoulis, M. Kafesaki, E.N. Economou, Negative-index materials new frontiers in optics, Adv. Mater. 18 (2006) 19411952.

[21] J. Zhou, Th. Koschny, M. Kafesaki, E.N. Economou, J.B. Pendry, C.M. Soukoulis, Saturation of the magnetic response of split-ring resonators at optical frequencies, Phys. Rev. Lett. 95 (2005) 223902.

[22] A.K. Azad, Y. Zhao, W. Zhang, M. He, Effect of dielectric properties of metals on terahertz transmission in subwavelength hole arrays, Opt. Lett. 31 (2006) 2637-2639.

[23] K.B. Alici, E. Ozbay, Low-temperature behavior of magnetic metamaterial elements, New J. Phys. 11 (2009) 043015. 